\title{
ARCS IN HYPERSPACES WHICH ARE NOT COMPACT
}

\author{
L. E. WARD, JR. ${ }^{1}$
}

Abstract. It has been known for many years that if $X$ is a metrizable continuum then $2^{X}$ (the space of closed subsets of $X$ ) and $C(X)$ (the subspace of connected members of $2^{X}$ ) are arcwise connected. These results are due to Borsuk and Mazurkiewicz [1] and J. L. Kelley [2], respectively. Quite recently M. M. McWaters [6] extended these theorems to the case of continua which are not necessarily metrizable, using Koch's arc theorem for partially ordered spaces [3], [8]. In this note we prove these results for certain noncompact spaces by means of a simple generalization of Koch's arc theorem.

1. Introduction. Recall that if $X$ is a topological space then $2^{X}$ denotes the space of nonempty closed subsets of $X$ with the Vietoris topology [7]. That is, if $U_{1}, U_{2}, \cdots, U_{n}$ are subsets of $X$ we write

$$
\begin{aligned}
\left\langle U_{1}, U_{2}, \cdots, U_{n}\right\rangle= & \left\{A \in 2^{x}: A \subset \bigcup_{i=1}^{n}\left\{U_{i}\right\}\right. \text { and } \\
& \left.\quad A \cap U_{i} \neq \varnothing \text { for each } i=1,2, \cdots, n\right\}
\end{aligned}
$$

and the family of all $\left\langle U_{1}, U_{2}, \cdots, U_{n}\right\rangle$ with $U_{i}$ open is a base for the open sets. The subspace of all closed and connected sets is denoted $C(X)$.

In this note our principal result is a partial extension of the recent theorem of McWaters [6], that if $X$ is an arbitrary continuum, then $2^{X}$ and $C(X)$ are arcwise connected.

TheOREM 1. If $X$ is a locally compact, locally connected and connected Hausdorff space, then $2^{X}$ is arcwise connected. If, in addition, $X$ is a normal space then $C(X)$ is arcwise connected.

At the end of the paper we give an example which shows that the hypothesis of local connectivity cannot be omitted.

It is a pleasure to express my appreciation to Professor Sam B. Nadler, Jr. who rekindled my interest in hyperspaces through several conversations and a lively correspondence.

Received by the editors January 5, 1970.

A MS 1969 subject classifications. Primary 5455; Secondary 0620, 5456.

Key words and phrases. Hyperspace, arc, partially ordered space.

1 This research was supported by a grant from the National Science Foundation. 
2. A theorem on partially ordered spaces. A partially ordered space is a topological space $S$ with a partial order $\Gamma$ which is a closed subset of $S \times S$. We treat the symbols $y \leqq x, y \in \Gamma x, x \in y \Gamma$ and $(y, x) \in \Gamma$ as synonyms. Of course $y<x$ means that $y \leqq x$ and $y \neq x$. A zero of a partially ordered space $S$ is an element $0 \in S$ such that $0 \Gamma=S$.

McWaters' proof of arcwise connectivity depended on showing that if $X$ is a continuum then $2^{X}$ and $C(X)$ can be regarded as partially ordered spaces which satisfy the following form of Koch's arc theorem [3], [8]: if $S$ is a compact partially ordered space with zero and if $\Gamma x$ is connected for each $x \in S$, then each nonzero element of $S$ is the supremum of an order arc containing the zero. For the applications of interest to us, a stronger version of Koch's theorem is required.

THEOREM 2. Let $S$ be a partially ordered space with zero, and suppose that for each nonzero $x \in S$ there exists $y<x$ such that if $y \leqq t \leqq x$ then $y \Gamma \cap \Gamma t$ is a continuum. If each nonempty chain of $S$ has an infimum then $S$ is arcwise connected.

Proof. Let 0 be the zero of $S$ and let $x \in S-\{0\}$. By hypothesis there exists $y<x$ such that $y \Gamma \cap \Gamma x$ is compact and $y \Gamma \cap \Gamma t$ is connected for each $t \in y \Gamma \cap \Gamma x$. Therefore, $y \Gamma \cap \Gamma x$ satisfies the hypotheses of Koch's arc theorem and there is an order arc whose supremum is $x$ and whose infimum is $y$. Let $A$ be the union of a maximal nest of order arcs each of which has $x$ for its supremum. By hypothesis $A$ has an infimum, and by a simple maximality argument that infimum is 0 . Thus each element of $S-\{0\}$ is joined to 0 by an arc.

3. Proof of the main result. We shall develop a series of lemmas which culminates in a proof of Theorem 1.

Lemma 1. If $X$ is a normal space then $C(X)$ is a closed subset of $2^{X}$.

A proof of Lemma 1 is given in $[5$, p. 139]. We define a relation $g$ on $2^{X}$ (the inclusion relation) by $(A, B) \in g$ if and only if $A \supset B$. Consistent with the notation for partial orders in $\$ 2$ we also write

$$
\mathscr{g} B=\left\{A \in 2^{x}:(A, B) \in \mathfrak{g}\right\}
$$

and $A \mathscr{G}$ is defined dually. Note that relative to the partial order $\mathscr{g}, X$ is a zero for $2^{X}$.

For an alternate proof of Lemma 2, see [4, p. 167].

Lemma 2. If $X$ is a regular space then $g$ is a closed subset of $2^{X} \times 2^{X}$.

Proof. If $(A, B) \in 2^{x} \times 2^{x}-g$ then there exists $b_{0} \in B-A$ and since $X$ is regular there are disjoint open sets $U$ and $V$ such that $b_{0} \in U$ and $A \subset V$. Note that $N(A)=\langle V\rangle$ is a neighborhood of $A$. If $A$ and $B$ have 
a point in common we set $N(B)=\langle U, X-A, V\rangle$ and otherwise $N(B)$ $=\langle U, X-A\rangle$. In either case $N(B)$ is a neighborhood of $B$ and $N(A) \times N(B)$ contains no member of $g$.

LEMMA 3. If $\mathfrak{X}$ is a nonempty nest which is a closed subset of $2^{X}$ or $C(X)$ then $\mathrm{Cl}(\cup \Re) \in \Re$.

Proof. Obviously $\mathrm{Cl}(\cup \Re) \in 2^{X}$ and if $\Re C C(X)$ then $\mathrm{Cl}(U \Re) \in C(X)$. If $\operatorname{Cl}\left(\cup_{\Re}\right) \in\left\langle U_{1}, U_{2}, \cdots, U_{n}\right\rangle$ where the $U_{i}$ are open subsets of $X$ then $U_{\mathscr{T}}$ meets each $U_{i}$ and hence there exists $N \in \mathscr{N}$ such that $N \in\left\langle U_{1}, U_{2}, \cdots, U_{n}\right\rangle$. Since $\Re$ is closed the lemma follows.

LEMma 4. If $X$ is a locally compact, locally connected and connected Hausdorff space and if $Y \in 2^{X}-\{X\}$ then there exists $Z \in g Y-\{Y\}$ such that if $R \in Z g \cap g Y$ then $Z g \cap g R$ is a continuum.

Proof. Since $Y \neq X$ there exists $y_{0} \in Y \cap \overline{X-Y}$, and since $X$ is locally connected there exists a continuum $N$ which is a neighborhood of $y_{0}$. Hence $Z=N \cup Y$ is a member of $g Y-\{Y\}$. Further if $R \in Z g \cap g Y$ (that is, if $R \in 2^{X}$ and $Z \supset R \supset Y$ ) then we can define $\phi: 2^{N} \rightarrow 2^{X}$ by $\phi(A)=A \cup R$. It is easy to see that $\phi$ is continuous [4, p. 106]; moreover the range of $\phi$ is precisely $Z g \cap g R$. Since $2^{N}$ is a continuum, so is $Z g \cap g R$.

Lemma 5. Let $X$ be a locally compact, locally connected, connected normal Hausdorff space. If $Y \in C(X)-\{X\}$ then there exists $Z$ $\in(g Y-\{Y\}) \cap C(X)$ such that if $R \in C(Z) \cap g Y$ then $C(R) \cap g Y$ is a continuum.

Proof. As in the proof of Lemma 4 there exists a continuum $N$ which meets both $Y$ and $X-Y$. In fact, we may assume that $N$ is a locally connected continuum. Let $Z=N \cup Y$ and define $\phi: 2^{N} \rightarrow 2^{X}$ by $\phi(A)=A \cup Y$. Again $\phi$ is continuous and since $2^{N}$ is compact it follows that $Z g \cap g Y$ is compact. If $R$ is a connected member of $Z g \cap g Y$ then by Lemma $2, R g$ is closed in $2^{x}$ and hence, by Lemma $1, C(R) \cap g Y$ is compact. Now suppose $C(R) \cap g Y$ is not connected. Then it is the union of disjoint closed sets $P$ and $Q$ and we may suppose $R \in P$. Since $Q$ is a compact partially ordered space it contains an $g$-minimal element $K$. (That is, $K$ is a member of $Q$ which is properly contained in no member of $Q$.) Then there are open subsets $U_{1}, U_{2}, \ldots, U_{n}$ of $X$ such that $K \in\left\langle U_{1}, U_{2}, \cdots, U_{n}\right\rangle$ and $\left\langle\bar{U}_{1}, \bar{U}_{2}, \cdots, \bar{U}_{n}\right\rangle \cap P$ is empty. Now choose $r \in R-K$; since $R$ is locally compact and connected and since $R-K$ has compact closure, there exists a continuum $B \subset R$ which contains $r$ and meets $K$. Let $U=U_{1} \cup U_{2} \cup \ldots \cup U_{n}$ and let $K_{1}$ be the closure of a component of $B \cap U$ which meets $K$. It 
follows that $K \varsubsetneqq K \cup K_{1} \subset R$ and $K \cup K_{1} \in\left\langle\bar{U}_{1}, \bar{U}_{2}, \cdots, \bar{U}_{n}\right\rangle$. But then $K \cup K_{1}$ is a member of $Q$ which contains $K$ properly, and this is a contradiction. This completes the proof that $C(R) \cap g Y$ is a continuum.

Theorem 1 now follows directly from Theorem 2 and the lemmas. If $X$ is a locally compact Hausdorff space then by Lemma $2,2^{x}$ is a partially ordered space. It has a zero, $X$, and by Lemma 3 each chain of $X$ has an infimum. If $X$ is connected and locally connected then by Lemma 4 the remaining hypotheses of Theorem 2 are satisfied. If $X$ is also a normal space then Lemma 5 can be invoked instead of Lemma 4 to apply Theorem 2 to $C(X)$.

If $X$ is not locally connected but satisfies all other hypotheses of Theorem 1 then it may happen that neither $2^{x}$ nor $C(X)$ is arcwise connected. To see this we recall an example from R. L. Wilder's book $[9$, p. 102]. In the Cartesian plane let $C=\{(-1, y): 0 \leqq y\}, L$ $=\{(1, y): 0 \leqq y\}$, let $P_{n}$ denote the line segment joining $(-n /(n+1), 0)$ to $(0, n)$ and let $Q_{n}$ denote the line segment joining $(0, n)$ to $(1,0)$. If we set

$$
X=C \cup L \cup \bigcup_{n=1}^{\infty}\left\{P_{n} \cup Q_{n}\right\}
$$

then $X$ is a locally compact, connected Hausdorff space which is not locally connected. Now let $U=\{(x, y) \in X:(x+1)(y+1)<1\}$ so that $U$ is an open set which contains $C$ and which meets each of the sets $P_{n}$. If $\alpha$ is an arc in $2^{X}$ whose endpoints are $C$ and $X$ and if $\beta$ is the closure of the component of $\alpha \cap\langle U\rangle$ which contains $C$, then $\beta$ is an arc whose endpoints are $C$ and some $B_{1} \in 2^{X}$ where $B_{1} \subset \bar{U}$. Consequently $B_{1} \cap P_{n} \cap \bar{U}$ is not empty, for some $n$. In the natural ordering of $\beta$ from $C$ to $B_{1}$ there is a first element which meets $P_{n} \cap \bar{U}$, say $B_{0}$. Let $V$ be an open subset of $X$ which contains $P_{n} \cap \bar{U}$ but is contained in the complement of $C$ and of each $P_{k}(k \neq n)$. Then $B_{0} \in\langle X, V\rangle$ and since $\langle X, V\rangle$ is open in $2^{X}$ there exists $B$ between $C$ and $B_{0}$ in the arc $\beta$ with $B \in\langle X, V\rangle$. Since $B \subset U$ it follows that $B$ meets $P_{n} \cap \bar{U}$, and this contradicts the properties of $B_{0}$.

\section{REFERENCES}

1. K. Borsuk and S. Mazurkiewicz, Sur l'hyperspace d'un continu, C. R. Soc. Sci. Varsovie 24 (1931), 149-152.

2. J. L. Kelley, Hyperspaces of a continuum, Trans. Amer. Math. Soc. 52 (1942), 23-36. MR 3, 315.

3. R. J. Koch, Arcs in partially ordered spaces, Pacific J. Math. 9 (1959), 723-728. MR $21 \# 7269$. 
4. K. Kuratowski, Topology. Vol. I, rev. ed., Academic Press, New York; PWN, Warsaw, 1966. MR $36 \# 839 ; 840$. 1968.

5. —, Topology. Vol. II, rev. ed., Academic Press, New York; PWN, Warsaw,

6. M. M. McWaters, Arcs, semigroups and hyperspaces, Canad. J. Math. 20 (1968), 1207-1210. MR $37 \# 6914$.

7. E. A. Michael, Topologies on spaces of subsets, Trans. Amer. Math. Soc. 71 (1951), 152-182. MR 13, 54.

8. L. E. Ward, Jr., Concerning Koch's theorem on the existence of arcs, Pacific J. Math. 15 (1965), 347-355. MR 31 \#6206.

9. R. L. Wilder, Topology of manifolds, Amer. Math. Soc. Colloq. Publ., vol. 32, Amer. Math. Soc., Providence, R. I., 1949. MR 10, 614.

University of Oregon, Eugene, Oregon 97403 